\title{
Nikon.
}

\section{Robotic Microscopy with the Nikon Ti2 for high-content analysis applications}

\author{
Robotic Microscopy-a combination of high-content screening methods-enables multivariate \\ experimental approaches with large cell populations and member-level sensitivity. Here we explore \\ how the new Nikon Ti2 line of inverted research microscopes is uniquely suited to Robotic Microscopy \\ applications, focusing on work utilizing induced pluripotent stem cells (iPSCs) as disease models in \\ drug screening.
}

The new Nikon Eclipse Ti2-E microscope features a 25-mm field of view (FOV) camera port and hardware-triggered control of native devices. These features may be applied to greatly increase the throughput of robotic microscopes and other high-content screening platforms.

\section{Key robotic microscope functions}

"Robotic Microscopy" is a term used to describe the synthesis of a number of technologies for automated imaging and analysis with high-throughput and information content, including the following:

- Automated sample translation for serial imaging - may include robotics for removing and replacing samples.

- Automated device control_of filter wheels, light sources, etc.

- Image analysis routines integrated in an experimental design.

- Multi-parametric detection, including multi-channel detection. Robotic Microscopy typically includes the capability to remove and replace multiple culture vessels, as well as custom software allowing for longitudinal studies of individual live cells in multiple populations.

Compared with pre-configured 'box' systems, robotic microscopes configured on traditional microscope stands provide a more flexible platform for users to exchange components for evolving needs. These components can include optics, filter wheels, detectors, emission splitters, and even advanced hardware for photostimulation, as well as confocal and super-resolution microscopy. A current robotic microscope design, configured on Nikon's Ti-E inverted microscope, is shown in Figure 1.

\section{Case Study - Robotic Microscopy of ALS disease models}

The work of Dr. Steven Finkbeiner at the Gladstone Institute of Neurological Disease, a pioneer in the field of Robotic Microscopy ${ }^{1-3}$,

John Allen

Nikon Instruments Inc., Melville, New York, USA. Correspondence should be addressed to jrallen@nikon.net.

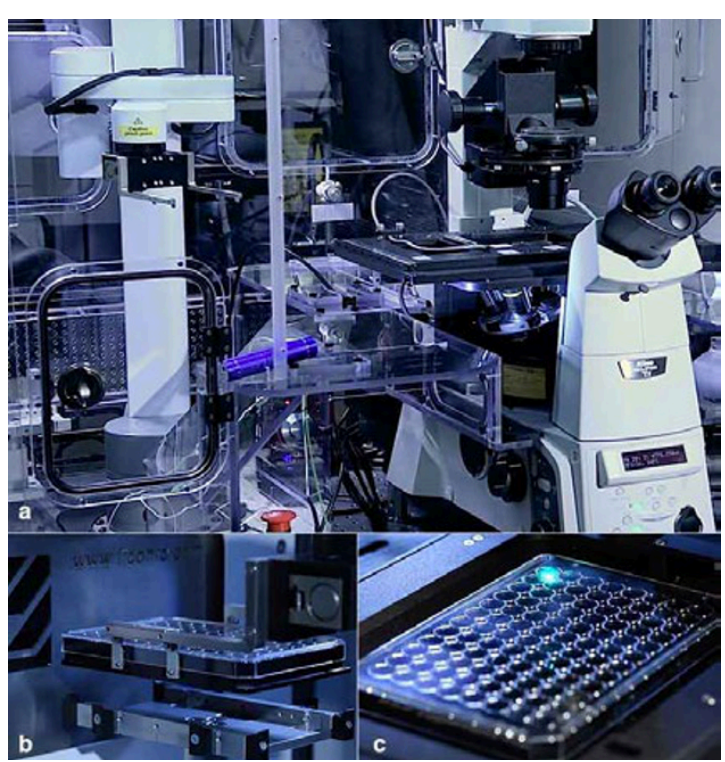

Figure 1 | Current robotic microscope design using a Nikon Ti-E inverted microscope. Future system designs utilizing the Nikon Ti2-E can potentially double the throughput of comparable systems. (a-c) Nikon Ti-E inverted microscope integrated with a robotic wellplate loader (a) for moving samples from incubator $(\mathbf{b})$ to microscope stage $(\mathbf{c})$. The system displayed in this figure belongs to the Finkbeiner laboratory.

utilizing iPSC disease models ${ }^{2}$ provides an excellent lens for understanding the potential of such systems for discovery and the importance of throughput.

Barmada et al. ${ }^{2}$ and Shaby et al. ${ }^{3}$ applied Robotic Microscopy to evaluate the treatment of amyotrophic lateral sclerosis (ALS), caused by mutations to TARDBP (also known as TDP43). High-content screening showed that increasing expression of both wild-type and mutant TDP-43 correlates with decreased cell survival.

Metabolic pulse chase (MPC) experiments have estimated the half- life of wild-type TDP-43 at 18 h (Fig. 2a,b), and this was confirmed by optical pulse labeling (OPL) experiments ${ }^{2}$ 
(Fig. 2d-f). OPL allows for longitudinal single-cell measurements over time, whereas MPC yields population-averaged data insensitive to parameters related to cell viability, morphology, etc. This example illustrates how imaging assays such as OPL can be designed to yield significantly greater information content than similar biochemical approaches. More recently, Shaby et al. ${ }^{3}$ applied Robotic Microscopy to screen a large drug library on ALS models.

The work of Barmada et al. ${ }^{2}$ and Shaby et al. ${ }^{3}$ was carried out using a Nikon TE-2000 microscope with Perfect Focus-critical for fast and accurate focusing and for reducing acquisition times and phototoxicity. The Nikon Ti2-E features the new Perfect Focus System 4, allowing for faster and more accurate focusing than ever before, especially important for multipoint imaging in high-content applications.
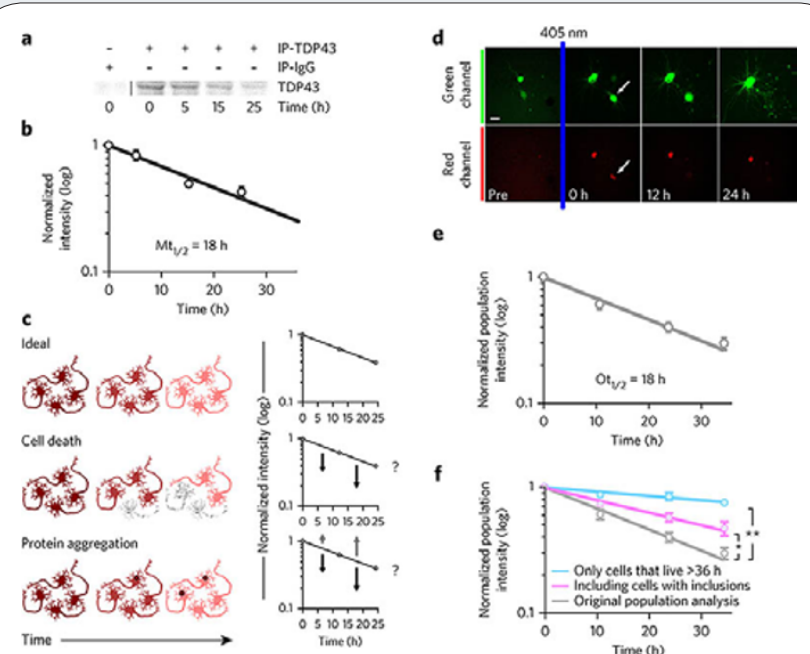

e
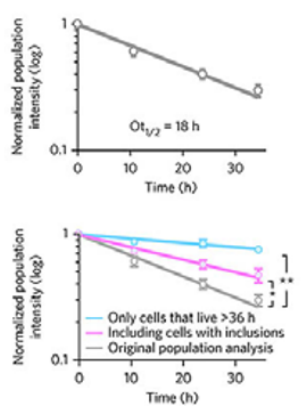

Figure 2 | Comparing MPC with OPL of TDP-43 using a robotic microscope. (a) MPC of TDP-43 from primary neurons, immunoprecipitated with anti-TDP-43 (IP-TDP43) or with nonspecific IgG antibodies (IP-IgG). (b) Determination of TDP-43 half-life from MPC data. (c) Noise sources in half-life measurements; cell death and protein aggregation confound estimates. (d) OPL of neurons expressing wild-type TDP-43 fused to Dendra2. (e) Half-life of wild-type TDP-43Dendra2 from OPL data. (f) Including cells with aggregates increased estimates of TDP-43 half-life. Figure reprinted with permission from ref. 2.

\section{Nikon's new Ti2-E inverted microscope for high-content} applications

Robotic microscopes are fundamentally throughput-limited by the number of images to be acquired. Nikon's Ti2 series features an unprecedented 25-mm-FOV camera port, providing a nearly twofold improvement in imaging area over the 18-mm FOV of its predecessor, the Ti-E. Current high-content imaging applications can require $\sim 4,500-8,000$ wells to be imaged per day ${ }^{1}$. The Ti2's larger FOV significantly reduces the number of images required to achieve the same results, providing an incredibly scalable and cost-effective solution.

Ti2's larger FOV is enabled through re-design of optical components and apertures in the light path, including larger filter cubes and tube lens. A fly-eye lens in the illumination path ensures even illumination across the FOV. The Ti2's large FOV is beneficial to large-format sensors, including Nikon's DS-Qi2 sCMOS (scientific complementary metal-oxide semiconductor) camera. In addition, smaller sensors benefit from this FOV, as even illumination yields more quantitative data and benefits image-stitching applications by minimizing post-processing steps, such as shading correction. The motorized, intelligent Ti2-E model features accessible TTL (transistor-transistor logic) signals for hardware-based triggering of native motorized components, allowing ultra-fast acquisition by minimizing latencies from software callbacks.

Automated imaging and analysis routines for high-content applications with the Ti2-E are easily customizable with Nikon's NIS- Elements HC software. The JOBS and General Analysis tools allow intelligent acquisition and conditional experiment flow-in which custom imaging experiments are determined by real-time analysis results - optimizing data collection. Figure 3 provides an example of a 96 well plate imaged with Nikon Ti2-E.

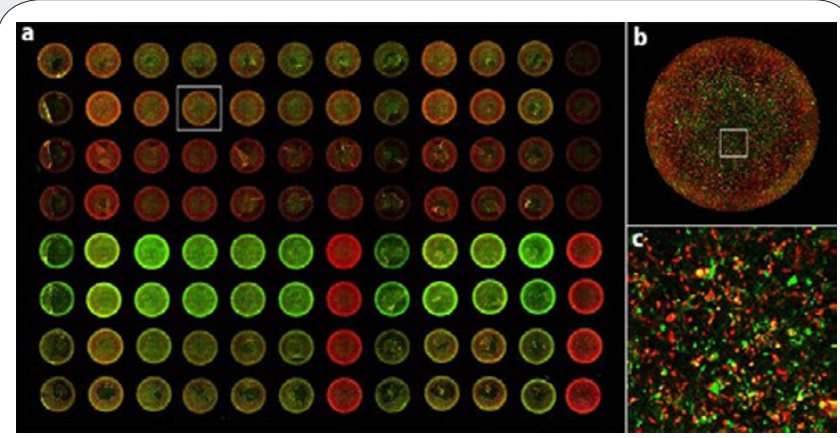

Figure 3 | 96-well plate containing iPSCs expressing green and red fluorescent protein markers imaged with the Nikon Ti2. (a) Whole 96-well plate imaged using a Nikon Eclipse Ti2-E microscope with a $4 \times$ objective and DS-Qi2 sCMOS camera. (b) Zoomed-in image of the well outlined in a. (c) Zoomed-in image of b. Sample courtesy of the Finkbeiner laboratory.

Future directions for robotic microscopy with the Nikon Ti2 Although super-resolution techniques have garnered much attention recently, the maturation of Robotic Microscopy provides a modern systems biology approach to increasingly complex problems, enabling the replacement of low-content biochemical assays with high-content imaging-based solutions. The Nikon Ti2's 25 mm field of view camera port and hardware triggering of native devices are key to developing higher throughput methods. Current work by the Finkbeiner lab and others emphasizes machine learning, which has the power to both make and test predictions in an iterative fashion and to incorporate non-imaging based information, such as genetic sequences and other clinical data, into high-content analysis.

1. Finkbeiner, S., Kassner, P. \& Frumkin, M. Neuron 86, 160-174 (2015). 2. Barmada, S.J. et al. Nat. Chem. Biol. 10,677-685 (2014). 3. Shaby, B.A. et al. Biometrics 72 936-944 (2016).

2. Barmada, S.J. et al. Nat. Chem. Biol. 10, 677-685 (2014)

3. Shaby, B.A. et al. Biometrics 72, 936-944 (2016).

This article was submitted to Nature Methods by a commercial organization and has not been peer reviewed. Nature Methods takes no responsibility for the accuracy or otherwise of the information provided. 